\title{
Singularity and decay estimates in superlinear problems via Liouville-type theorems. Part II: Parabolic equations
}

\author{
Peter Poláčik* \\ School of Mathematics, University of Minnesota \\ Minneapolis, MN 55455, USA \\ e-mail:polacik@math.umn.edu \\ Pavol Quittner ${ }^{\dagger}$ \\ Department of Applied Mathematics and Statistics, Comenius University, \\ Mlynská dolina, 84248 Bratislava, Slovakia \\ e-mail: quittner@fmph.uniba.sk \\ Philippe Souplet \\ Analyse, Géométrie et Applications, Institut Galilée, Université Paris-Nord, \\ 93430 Villetaneuse, France \\ e-mail: souplet@math.univ-paris13.fr
}

\begin{abstract}
In this paper, we study some new connections between parabolic Liouvilletype theorems and local and global properties of nonnegative classical solutions to superlinear parabolic problems, with or without boundary conditions. Namely, we develop a general method for derivation of universal, pointwise a priori estimates of solutions from Liouville-type theorems, which unifies and improves many results concerning a priori bounds, decay estimates and initial and final blow-up rates. For example, for the equation $u_{t}-\Delta u=u^{p}$ on a domain $\Omega$, possibly unbounded and not necessarily convex, we obtain initial and final blow-up rate estimates of the form $u(x, t) \leq C(\Omega, p)\left(1+t^{-\frac{1}{p-1}}+(T-t)^{-\frac{1}{p-1}}\right)$. Our method is based on rescaling arguments combined with a key "doubling" property, and it is facilitated by parabolic Liouville-type theorems for the whole
\end{abstract}

*Supported in part by NSF Grant DMS-0400702

${ }^{\dagger}$ Supported in part by VEGA Grant 1/3021/06 
space or the half-space. As an application of our universal estimates, we prove a nonuniqueness result for an initial boundary value problem.

\section{Introduction}

The aim of this paper is to study some new connections between parabolic Liouvilletype theorems and local and global properties of nonnegative classical solutions to superlinear parabolic problems. In the whole paper, the word "solution" always refers to "nonnegative solution", regardless of whether it is specifically mentioned. By a (nonlinear) Liouville-type theorem, we mean the statement of nonexistence of nontrivial bounded solutions defined for all negative and positive times on the whole space or on a half-space.

We develop a general method for obtaining pointwise, universal a priori estimates of solutions from Liouville-type theorems. The solutions that we consider are defined on an arbitrary spatial domain, without any prescribed initial conditions, but they may or may not satisfy boundary conditions. The word "universal" means that these estimates are independent of the solution itself (and even possibly of the domain).

We note that our approach works for both elliptic and parabolic problems, and that the elliptic aspects have been developed in the preceding part [28]. The method is based on rescaling arguments combined with a key "doubling" property (see Lemma 5.1 below). The doubling property is an extension of an idea of [20] (see Remark 5.3(a)). However, this powerful idea does not seem to have been fully exploited up to now, nor its wide applicability has been noticed.

Our approach enables us to unify and improve many results obtained in the last two decades on superlinear parabolic problems, concerning:

- boundedness and (universal) a priori estimates of global solutions;

- blow-up rates of nonglobal solutions;

- initial blow-up rates of local solutions;

- decay rates of global solutions of the Cauchy problem;

- spatial singularity estimates for local solutions.

Interestingly, from the heuristic point of view, Liouville-type theorems turn out to be equivalent to universal (initial or final) blow-up or decay estimates (cf. Remark 3.4(a)). Note, however, that some of the results previously obtained by different methods cannot still be entirely recovered here, due to the fact that the necessary Liouville theorems have not yet been proved in the optimal range of exponents.

On the other hand, we obtain some new Liouville-type theorems, especially for the case of half-spaces. Moreover, as another by-product of the method, we derive 
strong Liouville-type theorems from usual ones. By a strong Liouville-type theorem, we mean a statement of nonexistence of nontrivial solutions, bounded or not, defined for all negative and positive times on the whole space or on a half-space.

To show typical examples of our results, consider first the Cauchy problem associated with the model equation

$$
u_{t}-\Delta u=u^{p}
$$

where $p>1$ is less than a suitable exponent (see Section 2). We prove the initial and final blow-up rate estimate

$$
u(x, t) \leq C(n, p)\left(t^{-\frac{1}{p-1}}+(T-t)^{-\frac{1}{p-1}}\right), \quad x \in \mathbb{R}^{n}, \quad 0<t<T
$$

for any local solution on $\mathbb{R}^{n} \times(0, T)$, and the decay estimate

$$
u(x, t) \leq C(n, p) t^{-\frac{1}{p-1}}, \quad x \in \mathbb{R}^{n}, \quad t>0
$$

for any global solution on $\mathbb{R}^{n} \times(0, \infty)$. We stress the universality of the constant $C$. For the corresponding Dirichlet problem, in any smooth, possibly unbounded, domain $\Omega \subset \mathbb{R}^{n}$, these estimates take the form

$$
u(x, t) \leq C(\Omega, p)\left(1+t^{-\frac{1}{p-1}}+(T-t)^{-\frac{1}{p-1}}\right), \quad x \in \Omega, \quad 0<t<T,
$$

or

$$
u(x, t) \leq C(\Omega, p)\left(1+t^{-\frac{1}{p-1}}\right), \quad x \in \Omega, \quad t>0 .
$$

Note that this proves blow-up rates without assuming convexity of the domain, an assumption needed in the classical results of Giga and Kohn [17] (where, moreover, the constants $C$ depend on the solution).

Our method applies to more general superlinear parabolic equations such as the equation

$$
u_{t}-\Delta u=f(u) \text {, }
$$

where $f:[0, \infty) \rightarrow[0, \infty)$ is a continuous function satisfying a suitable growth condition. This includes in particular the model equation (1.1). We formulate most of our results in the context of equation (1.2), for further generalizations see Section 6 .

In some of our results, we consider radial solutions in symmetric domains. By a symmetric domain, we mean either the whole space $\mathbb{R}^{n}$, a ball $B_{R}=B(0, R) \subset \mathbb{R}^{n}$, an annulus $\Omega=\left\{x \in \mathbb{R}^{n} ; R_{1}<|x|<R_{2}\right\}$, or an exterior domain $\Omega=\left\{x \in \mathbb{R}^{n} ;|x|>R\right\}$, with $R>0, R_{2}>R_{1}>0$. We say that $u=u(x, t)$ is a radial function if $u(x, t)=$ $u(|x|, t)$ for each $t$.

The outline of the paper is as follows. In Section 2, we recall some known and present new parabolic Liouville-type theorems. Sections 3 and 4 contain our main results on universal (singularity and decay) estimates for equations (1.2) and (1.1), with and without prescribed boundary conditions, respectively. Section 5 contains the proofs of the singularity and decay estimates and of the strong Liouville-type theorems. An application (a nonuniqueness result for the Dirichlet initial boundary value problem associated with (1.1) with initial data in $L^{q}$ ) and generalizations can be found in Section 6 . 


\section{Parabolic Liouville-type theorems}

In order to state our results, we introduce the following exponents:

$$
p_{S}=p_{S}(n):= \begin{cases}\frac{n+2}{n-2}, & \text { if } n \geq 3, \\ \infty, & \text { if } n=1,2\end{cases}
$$

and

$$
p_{B}=p_{B}(n):= \begin{cases}\frac{n(n+2)}{(n-1)^{2}}, & \text { if } n \geq 2, \\ \infty, & \text { if } n=1 .\end{cases}
$$

Note that $p_{B}<p_{S}$ if $n \geq 2$.

The following two results will play an important role in our proof of singularity estimates. The first one is a direct consequence of [5] (see Remark 2.6(a) below).

Theorem A. Let $1<p<p_{B}(n)$. Then the equation

$$
u_{t}-\Delta u=u^{p}, \quad x \in \mathbb{R}^{n}, \quad t \in \mathbb{R},
$$

has no nontrivial (nonnegative) classical solution.

The proof in [5] is based on a modification of the technique of local, integral gradient estimates developed in [14] for elliptic problems (see also [6]). For $n=1$, a different proof is given in [27].

In the radial case, the following result was recently proved in [27] by using arguments of intersection-comparison with (sign-changing) stationary solutions (see [25] for an earlier partial result).

Theorem B. Let $1<p<p_{S}(n)$. Then equation (2.1) has no nontrivial (nonnegative) classical solution $u$, such that $u$ is radial and bounded.

Theorem $\mathrm{B}$ is optimal, since it is well-known that, for $n \geq 3$ and $p \geq p_{S}$, (2.1) admits positive stationary solutions which are radial and bounded. In view of Theorems A and B (and Theorem 2.3 below), one may conjecture that Theorem A should be true in the whole range $p<p_{S}$. However this remains an open problem.

Liouville theorems in a half-space $\mathbb{R}_{+}^{n}=\left\{x \in \mathbb{R}^{n}: x_{1}>0\right\}$ will also play an important role in our proof of singularity estimates for problems with boundary conditions. We will show the following.

Theorem 2.1. Let $p>1$.

(i) Assume $n \leq 2$, or $p<p_{B}(n-1)$ and $n \geq 3$. Then the problem

$$
\begin{aligned}
& \left.u_{t}-\Delta u=u^{p}, \quad x \in \mathbb{R}_{+}^{n}, \quad t \in \mathbb{R}, \quad\right\} \\
& \left.u=0, \quad x \in \partial \mathbb{R}_{+}^{n}, \quad t \in \mathbb{R}, \quad\right\}
\end{aligned}
$$

has no nontrivial (nonnegative) bounded classical solution. 
(ii) Assume $p<p_{B}(n)$. Then problem (2.2) has no nontrivial (nonnegative) classical solution.

Remarks 2.2. (a) Note that $p_{B}(n-1)>p_{S}(n)$ if $n \geq 3$.

(b) Up to now, the only available result of this kind in a half-space was the Fujita-type theorem from $[21,22]$ which states that (2.2) has no nontrivial solutions in $\mathbb{R}_{+}^{n} \times \mathbb{R}_{+}$if (and only if) $1<p \leq(n+3) /(n+1)$.

(c) The proof of Theorem 2.1 shows in fact the following:

(i) if the equation $u_{t}-\Delta u=u^{p}$ has no nontrivial bounded solution in $\mathbb{R}^{n-1} \times \mathbb{R}$ for given $p>1$ and $n \geq 2$, then (2.2) has no nontrivial bounded solution for those $p$ and $n$;

(ii) if the equation $u_{t}-\Delta u=u^{p}$ has no nontrivial bounded solution in $\mathbb{R}^{n} \times \mathbb{R}$ for given $p>1$ and $n \geq 1$, then (2.2) has no nontrivial solution for those $p$ and $n$.

Therefore, any improvement of Liouville theorems in the whole space would accordingly improve the half-space case. (Note of course that if the Liouville theorem is true in $\mathbb{R}^{n} \times \mathbb{R}$ for given $p>1$ and $n \geq 2$, then so is it in $\mathbb{R}^{n-1} \times \mathbb{R}$ : otherwise just consider $\tilde{u}\left(x_{1}, \ldots, x_{n-1}, x_{n}, t\right):=u\left(x_{1}, \ldots, x_{n-1}, t\right)$.)

(d) It was proved in [26] that equation (2.1) with $1<p<p_{S}$ has no nontrivial solution $u$ such that $|t|^{1 /(p-1)}\|u(t)\|_{\infty}$ is bounded as $t \rightarrow-\infty$. However, it does not seem possible to use this form of Liouville-type theorem to derive our results on universal singularity and decay estimates.

Finally, getting back to the radial case in the whole space, we can improve Theorem B by removing the boundedness assumption.

Theorem 2.3. Let $1<p<p_{S}(n)$. Then equation (2.1) has no nontrivial (nonnegative) classical, radial solution.

Theorems 2.1(ii) and 2.3 will be proved in Section 5. Theorem 2.1(i) is a consequence of Theorem A and the following theorem concerning the more general problem

$$
\left.\begin{array}{rlrl}
u_{t}-\Delta u & =f(u), & & x \in \mathbb{R}_{+}^{n}, t \in \mathbb{R}, \\
u & =0, & & x \in \partial \mathbb{R}_{+}^{n}, t \in \mathbb{R},
\end{array}\right\}
$$

where $f$ is a $C^{1}$-function.

Theorem 2.4. Assume $f:[0, \infty) \rightarrow \mathbb{R}$ is a $C^{1}$-function satisfying $f(0)=0$ and $f^{\prime}(0) \leq 0$. Then the following statements hold true. 
(c1) Each positive bounded solution $u$ of (2.3) is increasing in $x_{1}$ :

$$
\partial_{x_{1}} u(x, t)>0, \quad x \in \mathbb{R}_{+}^{n}, t \in \mathbb{R} .
$$

(c2) If there is a positive bounded solution of (2.3), then there exists a positive bounded solution of

$$
u_{t}-\Delta u=f(u), \quad x \in \mathbb{R}^{n-1}, t \in \mathbb{R} .
$$

For $n=1$, equation (2.4) should be understood as the ordinary differential equation $u_{t}=f(u)$.

The proofs of both statements (c1) and (c2) use extensions of arguments of [9] to parabolic equations. The idea to use these arguments was suggested to us by B. Sirakov. A straightforward modification of the proof below shows that (c1), (c2) hold for positive bounded solutions defined on $(-\infty, T)$ for some $T>0$.

Proof of Theorem 2.4. First we prove (c1). We use the following notation. For $\lambda>0$ let

$$
\mathbb{T}_{\lambda}=\left\{x \in \mathbb{R}^{n}: 0<x_{1}<\lambda\right\} .
$$

For a function $z$ defined on $\mathbb{R}_{+}^{n}$ let $z^{\lambda}$ and $V_{\lambda} z$ be functions on $\mathbb{T}_{\lambda}$ defined by

$$
\begin{aligned}
z^{\lambda}(x) & =z\left(2 \lambda-x_{1}, x^{\prime}\right), \\
V_{\lambda} z(x) & =z^{\lambda}(x)-z(x),
\end{aligned}
$$

where $x^{\prime}=\left(x_{2}, x_{3}, \ldots, x_{n}\right)$.

Let $u$ be a positive bounded solution of (2.3). Observe that for each $\lambda>0$, $v=V_{\lambda} u$ satisfies

$$
\begin{aligned}
v_{t}-\Delta v & =c^{\lambda}(x, t) v, & & x \in \mathbb{T}_{\lambda}, t \in \mathbb{R}, \\
v & =0, & & x_{1}=\lambda, x^{\prime} \in \mathbb{R}^{n-1}, t \in \mathbb{R}, \\
v & >0, & & x_{1}=0, x^{\prime} \in \mathbb{R}^{n-1}, t \in \mathbb{R},
\end{aligned}
$$

where

$$
c^{\lambda}(x, t)=\int_{0}^{1} f^{\prime}\left(u(x, t)+s\left(u^{\lambda}(x, t)-u(x, t)\right)\right) d s .
$$

Our goal is to prove that the statement

$$
(\mathrm{S})_{\lambda} \quad V_{\lambda} u(x, t) \geq 0, \quad x \in \mathbb{T}_{\lambda}, t \in \mathbb{R},
$$

holds for each $\lambda>0$. Once this done, the maximum principle applied to the above linear problem implies that we have in fact the strict inequality in $(S)_{\lambda}$ and the Hopf boundary principle then gives

$$
\left.2 \partial_{x_{1}} u(x, t)\right|_{x_{1}=\lambda}=-\left.\partial_{x_{1}} V_{\lambda} u(x, t)\right|_{x_{1}=\lambda}>0
$$

for each $\lambda>0$, proving $(\mathrm{c} 1)$.

We shall use the following lemma of Dancer [9]. 
Lemma 2.5. Given any positive constants $q, \lambda$ satisfying $\lambda^{-2} \pi^{2}>q$, there exists a smooth function $h$ on $\overline{\mathbb{T}}_{\lambda}$ such that

$$
\begin{aligned}
\Delta h+q h & =0, & & x \in \mathbb{T}_{\lambda}, \\
h(x) & >0, & & x \in \overline{\mathbb{T}}_{\lambda}, \\
h(x) & \rightarrow \infty, & & |x| \rightarrow \infty, x \in \overline{\mathbb{T}}_{\lambda} .
\end{aligned}
$$

Note that the function $h$ necessarily satisfies $h \geq \epsilon$ for some positive constant $\epsilon$. We first prove that $(S)_{\lambda}$ holds for $\lambda \approx 0$. Fix a positive constant $\gamma$ and set

$$
q:=\sup _{t \in \mathbb{R}, x \in \mathbb{R}_{+}^{n}} f^{\prime}(u(x, t))+\gamma .
$$

If $\lambda>0$ is sufficiently small, so that $\lambda^{-2} \pi^{2}>q$, we can apply Lemma 2.5 . With the resulting function $h$, we consider the problem satisfied by $w:=e^{\gamma t} v / h$, where $v=V_{\lambda} u$. A simple computation using (2.7), (2.9) shows that

$$
\begin{array}{rlr}
w_{t}-\Delta w-\frac{2 \nabla h}{h} \cdot \nabla w-\left(\gamma+c^{\lambda}(x, t)-q\right) w=0, & x \in \mathbb{T}_{\lambda}, t \in \mathbb{R}, \\
w & \geq 0, \\
w(x, t) & \rightarrow 0, & x \in \partial \mathbb{T}_{\lambda}, t \in \mathbb{R},
\end{array}
$$

The choice of $q$ implies that $\gamma+c^{\lambda}-q \leq 0$ in $\mathbb{T}_{\lambda} \times \mathbb{R}$. Applying the maximum principle on $\mathbb{T}_{\lambda} \times\left(t_{0}, t\right)$, for any $t_{0}<t$, we obtain

$$
\sup _{x \in \mathbb{T}_{\lambda}} w^{-}(x, t) \leq \sup _{x \in \mathbb{T}_{\lambda}} w^{-}\left(x, t_{0}\right),
$$

where $z^{-}$stands for the negative part of $z: z^{-}=-\min (z, 0)$. For $v$ the above inequality means

$$
\sup _{x \in \mathbb{T}_{\lambda}} \frac{v^{-}(x, t)}{h(x)} \leq e^{-\gamma\left(t-t_{0}\right)} \sup _{x \in \mathbb{T}_{\lambda}} \frac{v^{-}\left(x, t_{0}\right)}{h(x)} .
$$

In view of boundedness of $v=V_{\lambda} u$, letting $t_{0} \rightarrow-\infty$ we obtain that $v \geq 0$ everywhere, hence $(\mathrm{S})_{\lambda}$ holds.

In the next step we denote

$$
\lambda_{0}=\sup \left\{\mu>0:(\mathrm{S})_{\lambda} \text { holds for all } \lambda \in(0, \mu)\right\} .
$$

As proved above, $\lambda_{0}>0$. We now show by contradiction that $\lambda_{0}=\infty$. Assume $\lambda_{0}<\infty$. Then there is a sequence $\lambda_{k} \geq \lambda_{0}$ such that $\lambda_{k} \rightarrow \lambda_{0}$ and the set

$$
Z_{k}:=\left\{(x, t) \in \mathbb{T}_{\lambda_{k}} \times \mathbb{R}: V_{\lambda_{k}} u(x, t)<0\right\}
$$

is nonempty. Set

$$
\begin{aligned}
m_{k}:=\sup \left\{u\left(y_{1}, x^{\prime}, t\right):\right. & y_{1} \in\left(0, \lambda_{k}\right), x^{\prime} \in \mathbb{R}^{n-1}, t \in \mathbb{R}, \text { and } \\
& \text { there exists } \left.x_{1} \in\left(0, \lambda_{k}\right) \text { such that }\left(x_{1}, x^{\prime}, t\right) \in Z_{k}\right\} .
\end{aligned}
$$

We consider the following two possibilities. 
(a) $m_{k} \rightarrow 0$

(b) passing to a subsequence we have $m_{k} \geq \varepsilon_{0}$ for some $\varepsilon_{0}>0$.

First assume that (b) holds. Then there are sequences $x_{1}^{k}, y_{1}^{k} \in\left(0, \lambda_{k}\right), z^{k} \in \mathbb{R}^{n-1}$, $t^{k} \in \mathbb{R}$ such that $V_{\lambda_{k}} u\left(x_{1}^{k}, z^{k}, t^{k}\right)<0$ and $u\left(y_{1}^{k}, z^{k}, t^{k}\right) \geq \varepsilon_{0}$. We may assume that $x_{1}^{k} \rightarrow a$ and $y_{1}^{k} \rightarrow b$ for some $a, b \in\left[0, \lambda_{0}\right]$. Consider the functions

$$
u_{k}(x, t):=u\left(x_{1}, x^{\prime}+z^{k}, t+t^{k}\right), \quad x=\left(x_{1}, x^{\prime}\right) \in \mathbb{R}^{n}, t \in \mathbb{R} .
$$

Each of them is a positive solution of (2.3) satisfying $V_{\lambda_{k}} u_{k}\left(x_{1}^{k}, 0,0\right)<0, u_{k}\left(y_{1}^{k}, 0,0\right) \geq$ $\varepsilon_{0}$ and $V_{\lambda_{0}} u_{k} \geq 0$ in $\mathbb{T}_{\lambda_{0}} \times \mathbb{R}$ (the last inequality follows from the definition of $\lambda_{0}$ and continuity). Moreover, the sequence $u_{k}$ is uniformly bounded. Using standard parabolic estimates, one shows that if $u_{k}$ is replaced by a subsequence, then it converges in $C_{\text {loc }}^{2,1}\left(\mathbb{R}^{n+1}\right)$ to a nonnegative solution $\tilde{u}$ of $(2.3)$. The above properties of $u_{k}$ imply that $V_{\lambda_{0}} \tilde{u}(a, 0,0) \leq 0, \tilde{u}(b, 0,0) \geq \varepsilon_{0}$, and $V_{\lambda_{0}} \tilde{u} \geq 0$ in $\mathbb{T}_{\lambda_{0}} \times \mathbb{R}$. Since $\tilde{u}$ is nontrivial and $f(0)=0$ the maximum principle implies that $\tilde{u}$ is positive everywhere. Consequently, $\tilde{v}:=V_{\lambda_{0}} \tilde{u}$ solves the corresponding problem (2.7) with $\lambda=\lambda_{0}$ and therefore $\tilde{v}>0$ in $\mathbb{T}_{\lambda_{0}} \times \mathbb{R}$. It follows in particular that necessarily $a=\lambda_{0}$. By the Hopf principle,

$$
2 \tilde{u}_{x_{1}}\left(\lambda_{0}, 0,0\right)=-\left.\partial_{x_{1}} V_{\lambda_{0}} \tilde{u}\left(x_{1}, 0,0\right)\right|_{x_{1}=\lambda_{0}}>0 .
$$

Consequently, $\tilde{u}_{x_{1}}\left(x_{1}, 0,0\right)$ is bounded below by a positive constant on an interval around $\lambda_{0}$ and this remains valid if $\tilde{u}$ is replaced by $u_{k} \approx u$. That is, there is $\delta>0$ such that

$$
\partial_{x_{1}} u\left(x_{1}, z^{k}, t^{k}\right)=\partial_{x_{1}} u_{k}\left(x_{1}, 0,0\right)>0, \quad x_{1} \in\left[\lambda_{0}-\delta, \lambda_{0}+\delta\right],
$$

for all sufficiently large $k$. However, since $2 \lambda_{k}-x_{1}^{k}>x_{1}^{k}$ both belong to $\left[\lambda_{0}-\delta, \lambda_{0}+\delta\right]$ for large $k,(2.15)$ contradicts the assumption that $V_{\lambda_{k}} u\left(x_{1}^{k}, z^{k}, t^{k}\right)<0$.

We have shown that (b) leads to a contradiction. Assume now that (a) holds. Consider the problem (2.7) with $\lambda=\lambda_{k}$ and $k$ sufficiently large. We are going to apply the maximum principle on the set $Z_{k}$ (assumed to be nonempty). The boundary conditions in (2.7) imply that $v=V_{\lambda_{k}} u=0$ on $\partial Z_{k}$. Next observe that property (a), in conjunction with $(2.8), f^{\prime}(0) \leq 0$ and the definition of $m_{k}$, implies that for

$$
\tilde{q}_{k}:=\sup _{(x, t) \in Z_{k}} c^{\lambda_{k}}(x, t)
$$

we have

$$
\limsup _{k \rightarrow \infty} \tilde{q}_{k} \leq 0 \text {. }
$$

Fix $k$ so large that $q:=\tilde{q}_{k}+\gamma<\lambda_{k}^{-2} \pi^{2}\left(\approx \lambda_{0}^{-2} \pi^{2}\right)$, where $\gamma$ is some positive constant and set $\lambda=\lambda_{k}$. Apply Lemma 2.5 and let $h$ be the resulting function. As in our arguments above, $w:=e^{\gamma t} v / h$ satisfies problem (2.11). This time we know that $\gamma+$ $c^{\lambda}-q \leq 0$ on $Z_{k}$ only. However, since $v$ vanishes on $\partial Z_{k}$, we can still apply the 
maximum principle on $Z_{k}$ to conclude that (2.12) holds and, consequently, that $v \geq 0$ in $Z_{k}$. This of course contradicts the definition of $Z_{k}$. Thus possibility (a) leads to a contradiction, too, which proves that $\lambda_{0}=\infty$.

We have completed the proof of $(\mathrm{c} 1)$.

To prove (c2), let $u$ be a positive bounded solution of (2.3). For $k=1,2, \ldots$ consider the functions

$$
u_{k}\left(x_{1}, x^{\prime}, t\right):=u\left(x_{1}+k, x^{\prime}, t\right), \quad\left(x_{1}, x^{\prime}, t\right) \in(-k, \infty) \times \mathbb{R}^{n} \times \mathbb{R} .
$$

Each of them solves the equation $u_{t}=\Delta u+f(u)$ on its domain. Since the sequence is uniformly bounded, using parabolic estimates one shows that a subsequence of $u_{k}$ converges uniformly on each compact to a bounded nonnegative solution $\tilde{u}$ of $u_{t}=\Delta u+f(u)$ on $\mathbb{R}^{n+1}$. From the monotonicity of $u$ proved in (c1), we further conclude that $\tilde{u}$ is positive and independent of $x_{1}$. This proves (c2).

Remarks 2.6. (a) In [5], for any positive solution $u$ of (1.1) on $\{x:|x|<2\} \times(-2,2)$, the author proved the integral estimate:

$$
\int_{-1}^{1} \int_{|x|<1} u^{r} d x d t \leq C(n, p),
$$

with $r=2 p>(n+2)(p-1) / 2$. A pointwise (initial blow-up rate) estimate was then deduced from (2.16), by using the Harnack inequality for the heat equation with a potential, and such estimate implies the Liouville-type Theorem A. However, we observe that Theorem A can be deduced directly from estimate (2.16) by the following simple homogeneity argument. Fix $R>0$. If $u$ is a solution of (2.1), then so is $v(x, t):=R^{2 /(p-1)} u\left(R x, R^{2} t\right)$. If follows from (2.16) applied to $v$ that

$$
\begin{aligned}
\int_{-R^{2}}^{R^{2}} \int_{|y|<R} u^{r}(y, s) d y d s & =R^{n+2} \int_{-1}^{1} \int_{|x|<1} u^{r}\left(R x, R^{2} t\right) d x d t \\
& =R^{n+2-2 r /(p-1)} \int_{-1}^{1} \int_{|x|<1} v^{r}(x, t) d x d t \leq C(n, p) R^{n+2-2 r /(p-1)} .
\end{aligned}
$$

Since $r>(n+2)(p-1) / 2$, by letting $R \rightarrow \infty$, we conclude that $\int_{-\infty}^{\infty} \int_{\mathbb{R}^{n}} u^{r}(y, s) d y d s=$ 0 , hence $u \equiv 0$.

(b) In the case $n=1$, Theorem 2.1(i) can be proved alternatively by using intersection-comparison arguments similar to those in [27].

\section{Problems without boundary conditions}

We consider equation (1.2) for $x \in \Omega$ and $t \in(0, T)$, where $\Omega$ is an arbitrary domain in $\mathbb{R}^{n}$ and $T>0$. By a solution we mean a classical solution $u \in C^{2,1}(\Omega \times(0, T))$. We use the usual distance function in $\operatorname{dist}(x, \partial \Omega)$, which we assume to equal $\infty$ if $\Omega=\mathbb{R}^{n}$. 
Theorem 3.1. Let $p>1, T>0, \Omega$ be an arbitrary domain of $\mathbb{R}^{n}, f:[0, \infty) \rightarrow \mathbb{R}$ be a continuous function such that

$$
\lim _{u \rightarrow \infty} u^{-p} f(u)=\ell \in(0, \infty)
$$

and $u$ be a (nonnegative) solution of (1.2) on $\Omega \times(0, T)$. Assume that either

$$
p<p_{B}, \quad \text { or } p<p_{S}, \Omega \text { is symmetric and } u \text { is radial. }
$$

(i) Then there holds

$$
u(x, t) \leq C\left(1+t^{-\frac{1}{p-1}}+(T-t)^{-\frac{1}{p-1}}+\operatorname{dist}^{-\frac{2}{p-1}}(x, \partial \Omega)\right), \quad x \in \Omega, \quad 0<t<T,
$$

with a constant $C=C(n, f)>0$, independent of $\Omega, T$ and $u$.

(ii) If $f(u)=u^{p}$, then conclusion (3.3) can be replaced by

$$
u(x, t) \leq C(n, p)\left(t^{-\frac{1}{p-1}}+(T-t)^{-\frac{1}{p-1}}+\operatorname{dist}^{-\frac{2}{p-1}}(x, \partial \Omega)\right), \quad x \in \Omega, \quad 0<t<T .
$$

As immediate consequences of Theorem 3.1 in the case of $\mathbb{R}^{n}$, we obtain a universal decay rate for all global solutions of $(1.1)$ in $\mathbb{R}^{n} \times(0, \infty)$ and universal initial and final blow-up rates for solutions of $(1.2)$ in $\mathbb{R}^{n} \times(0, T)$.

Corollary 3.2. Let $p>1$ and $u$ be a global (nonnegative) solution of (1.1) on $\mathbb{R}^{n} \times$ $(0, \infty)$. Assume (3.2). Then there holds

$$
u(x, t) \leq C(n, p) t^{-\frac{1}{p-1}}, \quad x \in \mathbb{R}^{n}, \quad t>0 .
$$

Corollary 3.3. Let $p>1, T>0$ and $u$ be a (nonnegative) solution of (1.2) on $\mathbb{R}^{n} \times(0, T)$. Assume (3.1), (3.2).

(i) Then there holds

$$
u(x, t) \leq C(n, f)\left(1+t^{-\frac{1}{p-1}}+(T-t)^{-\frac{1}{p-1}}\right), \quad x \in \mathbb{R}^{n}, \quad 0<t<T .
$$

(ii) If $f(u)=u^{p}$, then conclusion (3.6) can be replaced by

$$
u(x, t) \leq C(n, p)\left(t^{-\frac{1}{p-1}}+(T-t)^{-\frac{1}{p-1}}\right), \quad x \in \mathbb{R}^{n}, \quad 0<t<T .
$$

Corollary 3.2 in the case $p<p_{B}$ is a direct consequence of [5]. The radial case for $p<p_{S}$ is new. Besides [5], this partially improves various known results. In [21], the same decay rate was obtained for global solutions of the Cauchy problem for all $p<p_{S}$, but under the strong assumption that the initial data $u_{0}$ has Gaussian decay at $\infty$ (and with a constant $C$ depending on $u_{0}$ ). In [34], for $p<p_{S}$ and $u_{0} \in L^{2} \cap L^{\infty}$, the decay of global solutions was shown, but with no determined decay rate. In [25], the universal decay rate (3.5) was shown for $n \leq 3$ and $p<p_{S}$, but only for radial 
solutions which are nonincreasing in $|x|$. Finally in [27], for $p<p_{S}$, the decay of global solutions was shown (without definite rate) when $u_{0} \in L^{\infty}$ and $u$ is radial or $n=1$.

Corollary 3.3 for $p<p_{B}$ and $f(u)=u^{p}$ is also a direct consequence of [5]. The other cases are new. As for final blow-up rates for the Cauchy problem with $p<p_{S}$ and $f$ satisfying (3.1), the classical result of [17] shows that $u(x, t) \leq C(T-t)^{-1 /(p-1)}$ in $\mathbb{R}^{n} \times(0, T)$, but with a constant depending on the initial data $u_{0}$. The universal estimate (3.7) for $p<(n+2) / n$ is a consequence of [1] (see also [2,3]). In [25], it was shown for $f(u)=u^{p}, p<p_{S}$ and $n \leq 3$, but only for radial, radially decreasing solutions.

Remarks 3.4. (a) The conclusions of Theorem 3.1 and Corollaries 3.2 and 3.3 remain true if we replace assumption (3.2) by the assumption that equation (2.1) does not admit any bounded nontrivial solution. Conversely, it is clear that any of the estimates (3.4), (3.5) or (3.7) implies the nonexistence of nontrivial (even unbounded) solutions to (2.1). Therefore, we see that all these properties are essentially equivalent (see [28] for a similar phenomenon in the case of elliptic problems).

(b) The assumption $p<p_{S}$ in the above results cannot be improved, in general, due to the existence of nontrivial bounded (radial) solutions of $\Delta u+u^{p}=0$ in $\mathbb{R}^{n}$ for $p \geq p_{S}$. For $n \geq 11$ and $p$ larger than a certain exponent $p^{*}(n)\left(>p_{S}\right)$, it has even been shown [29] that unbounded global (classical) solutions of (1.1) in $\mathbb{R}^{n} \times[0, \infty)$ exist for some bounded initial data. On the other hand, if we consider the radial case and we restrict ourselves to symmetric domains $\Omega$ that do not contain the origin then Theorem 3.1 (and Theorem 4.1 below) remain true for any $p>1$. This follows from the proofs of those theorems.

(c) Consider the case $f(u)=u^{p}$ and $\Omega=\mathbb{R}^{n}$. It is well-known (for any $p>1$ ) that if $u$ ceases to exist (in the classical sense) at $t=T$, then the blow-up rate satisfies $\sup _{\mathbb{R}^{n}} u(\cdot, t) \geq C(p)(T-t)^{-1 /(p-1)}$, which shows the optimality of estimate (3.7) on $(T / 2, T)$. As for the decay rate in (3.5) and the initial blow-up rate (i.e. (3.7) on $(0, T / 2))$, they are optimal for $(n+2) / n<p<p_{S}$, due to the existence of positive (radial) self-similar solutions of the form $t^{-1 /(p-1)} f\left(|x| t^{-1 / 2}\right)[19]$. When $p \leq(n+2) / n$, there are no global solutions in $\mathbb{R}^{n} \times[0, \infty)$ due to the well-known Fujita result, but the order of the (optimal) initial blow-up rate for local solutions is then $t^{-n / 2}$ [5]. More precisely, [5] implies $u(x, t) \leq C(n, p) T^{(n / 2)-1 /(p-1)} t^{-n / 2}$ in $\mathbb{R}^{n} \times(0, T / 2]$.

(d) Estimate (3.3) can be expressed in a more concise equivalent form by using the parabolic distance

$$
d_{P}((x, t),(y, s)):=|x-y|+|t-s|^{1 / 2} .
$$

Namely, denote $D=\Omega \times(0, T), \partial D=(\partial \Omega \times[0, T]) \cup(\bar{\Omega} \times\{0, T\})$ the topological (not parabolic) boundary of $D$ in $\mathbb{R}^{n+1}$, and $d_{P}((x, t), \partial D)=\inf _{(y, s) \in \partial D} d_{P}((x, t),(y, s))$. Then estimate (3.3) can be restated as

$$
u(x, t) \leq C\left(1+d_{P}^{-\frac{2}{p-1}}((x, t), \partial D)\right), \quad(x, t) \in D .
$$


In fact, in the nonradial case we will prove this estimate for any domain $D \subset \mathbb{R}^{n+1}$, not necessarily of the form $\Omega \times(0, T)$. In the radial case we will assume $D=\Omega \times\left(T_{1}, T_{2}\right)$, where $\Omega$ is symmetric and $-\infty \leq T_{1}<T_{2} \leq \infty$.

Recall that the typical singularity estimate in the elliptic case (cf. [28]) takes the form

$$
u(x) \leq C\left(1+\operatorname{dist}^{-\frac{2}{p-1}}(x, \partial \Omega)\right), \quad x \in \Omega .
$$

Formula (3.8) thus allows us to state the elliptic and parabolic versions of our results in a unified and natural way.

(e) Let $d_{P}$ be as in the previous remark and let $D$ be any domain in $\mathbb{R}^{n+1}$ (or, in the radial case, $D=\Omega \times\left(T_{1}, T_{2}\right)$, where $\Omega$ is symmetric and $\left.-\infty \leq T_{1}<T_{2} \leq \infty\right)$. In the proof of Theorem 3.1 we will prove stronger estimates than announced:

$$
u(x, t)+|\nabla u(x, t)|^{\frac{2}{p+1}} \leq C\left(1+d_{P}^{-\frac{2}{p-1}}((x, t), \partial D)\right), \quad(x, t) \in D,
$$

instead of (3.3), and

$$
u(x, t)+|\nabla u(x, t)|^{\frac{2}{p+1}} \leq C d_{P}^{-\frac{2}{p-1}}((x, t), \partial D), \quad(x, t) \in D,
$$

instead of (3.4). If $f$ is Hölder continuous then an obvious modification of the proof guarantees estimates for the second spatial derivatives and the first time derivative. More precisely, one obtains the estimate

$$
u+|\nabla u|^{\frac{2}{p+1}}+\max _{i, j}\left|u_{x_{i} x_{j}}\right|^{\frac{1}{p}}+\left|u_{t}\right|^{\frac{1}{p}} \leq C\left(1+d_{P}^{-\frac{2}{p-1}}((x, t), \partial D)\right), \quad(x, t) \in D,
$$

instead of (3.3) and analogously for (3.4).

(f) Similarly as in [28, Theorem 2.3], Theorem 3.1 can be used in order to establish spatial decay of solutions of (1.1) in the case of exterior (spatial) domains. For example, if $p<p_{B}$ and $u$ is a (nonnegative) solution of (1.1) in $\left\{x \in \mathbb{R}^{n}:|x|>R\right\} \times \mathbb{R}$ then

$$
u(x, t) \leq C(n, p)|x|^{-\frac{2}{p-1}}, \quad|x|>2 R, t \in \mathbb{R} .
$$

Remark (b) above guarantees that this bound remains true for any $p>1$ provided $u$ is radial.

\section{Problems with boundary conditions}

We now consider the boundary value problem:

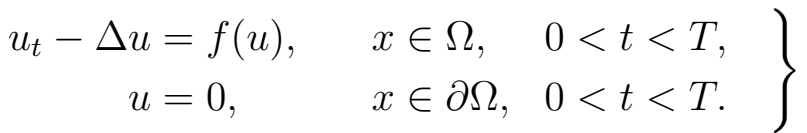

Here $\Omega$ is a (possibly unbounded, uniformly $C^{2}$ ) smooth domain in $\mathbb{R}^{n}$. By a solution of (4.1), we mean a classical solution $u \in C^{2,1}(\Omega \times(0, T)) \cap C(\bar{\Omega} \times(0, T))$. 
Theorem 4.1. Let $p>1,0<T \leq \infty, f:[0, \infty) \rightarrow \mathbb{R}$ be a continuous function satisfying (3.1), and $u$ be a (nonnegative) solution of (4.1). Assume that either

$$
p<p_{B}, \quad \text { or } p<p_{S}, \Omega \text { is symmetric and } u \text { is radial. }
$$

(i) If $T<\infty$, then there holds

$$
u(x, t) \leq C\left(1+t^{-\frac{1}{p-1}}+(T-t)^{-\frac{1}{p-1}}\right), \quad x \in \Omega, \quad 0<t<T,
$$

where $C=C(f, \Omega)$.

(ii) If $u$ is global (i.e. $T=\infty$ ), then we have

$$
u(x, t) \leq C\left(1+t^{-\frac{1}{p-1}}\right), \quad x \in \Omega, \quad t>0,
$$

where $C=C(f, \Omega)$.

We first point out that, even letting the universality of the constant $C$ aside, Theorem 4.1 improves the known results concerning final blow-up rate estimates of the form

$$
u(x, t) \leq C(T-t)^{-1 /(p-1)}, \quad x \in \Omega, \quad 0<t<T .
$$

Indeed, estimate (4.4) with $C$ depending on the solution $u$ was known before for $f(u)=u^{p}, p<p_{S}$, and for all blowing-up positive solutions, but under the assumption that $\Omega$ is convex $[17,18]$, or in general domains, but only for $p \leq(n+3) /(n+1)$ [11]. If one restricts to particular classes of solutions, (4.4) is known to be true for all $p>1$, in general bounded domains if $u_{t} \geq 0$ [13], or in an annulus if $u$ is radial [24]. Theorem 4.1 implies (4.4) in general domains whenever $p<p_{B}$ (greater than $(n+3) /(n+1))$.

Theorem 4.1 also provides universal bounds for global solutions (away from $t=0$ ), as well as universal estimates for the initial and final blow-up rates of local solutions. For general bounded domains, such results have been obtained (by completely different methods) in [33] for $f(u)=u^{p}$ under the assumption $p<p_{S}$ if $n \leq 4$, and $p<$ $(n-1) /(n-3)\left(<p_{B}\right)$ if $n \geq 5$. However, while we here find $1 /(p-1)$ as initial blowup rate exponent, no explicit value was obtained in [33]. In fact, the exponent $1 /(p-1)$ is optimal when $(n+3) /(n+1)<p<p_{S}$, but not when $1<p<(n+3) /(n+1)$. Indeed it was shown in [33] that the optimal initial blow-up rate in that range is $t^{-(n+1) / 2}$ (note the difference with the Cauchy problem, cf. Remark 3.4(c)). Earlier results on universal bounds for bounded domains were proved in $[12,31]$. For more general nonlinearities $f(u)$, some results without assumption of power behavior at infinity (but with relatively slow growth) can be found in $[12,33]$.

Finally, let us recall that an a priori estimate of global solutions in bounded domains is known in the full range $1<p<p_{S}$ [16] (and for sign-changing solutions as well [30]). In these results, the constants depend on the sup norm of the initial data. Recall also that universal bounds fail for sign-changing solutions (see e.g. [12]). 
In the case of the model equation (1.1) in a half-space, we have a stronger estimate which in particular yields a universal time decay of global solutions.

Theorem 4.2. Assume $1<p<p_{B}, 0<T<\infty$ (respectively $T=\infty$ ), and let $u$ be a (nonnegative) solution of (4.1) with $f(u)=u^{p}$ and $\Omega=\mathbb{R}_{+}^{n}$. Then there holds

$$
u(x, t) \leq C(n, p)\left(t^{-\frac{1}{p-1}}+(T-t)^{-\frac{1}{p-1}}\right), \quad x \in \mathbb{R}_{+}^{n}, \quad 0<t<T,
$$

respectively

$$
u(x, t) \leq C(n, p) t^{-\frac{1}{p-1}}, \quad x \in \mathbb{R}_{+}^{n}, \quad t>0 .
$$

Remarks 4.3. (a) Theorem 4.1 remains true under the assumption that (2.1) does not admit any bounded nontrivial solution (instead of assumption (4.2)). In particular, if one were able to prove Theorem A up to $p<p_{S}$, this would imply universal estimates for problem (4.1) in the optimal range $1<p<p_{S}$ (without symmetry restrictions).

(b) For monotone in time solutions, the symmetry assumption in Theorem 4.1 can be removed (and the conclusion strengthened). Assume $p<p_{S},(3.1)$, and let $u$ be a solution of (4.1), or of (1.2) if $\Omega=\mathbb{R}^{n}$, such that $u_{t} \geq 0$. Then one can prove (see Remark 5.3(b)) that

$$
u(x, t) \leq C(\Omega, f)\left(1+(T-t)^{-1 /(p-1)}\right), \quad x \in \Omega, \quad 0<t<T,
$$

and that

$$
u(x, t) \leq C(n, p)(T-t)^{-1 /(p-1)}, \quad x \in \mathbb{R}^{n}, \quad 0<t<T
$$

if $\Omega=\mathbb{R}^{n}$ and $f(u)=u^{p}$.

(c) A similar improvement as in Remark 3.4(e) is valid for problems with boundary conditions as well.

\section{Proof of universal a priori estimates}

A key-ingredient in our proofs is the following Lemma from [28].

Lemma 5.1. Let $(X, d)$ be a complete metric space and let $\emptyset \neq D \subset \Sigma \subset X$, with $\Sigma$ closed. Set $\Gamma=\Sigma \backslash D$. Finally let $M: D \rightarrow(0, \infty)$ be bounded on compact subsets of $D$ and fix a real $k>0$. If $y \in D$ is such that

$$
M(y) \operatorname{dist}(y, \Gamma)>2 k,
$$

then there exists $x \in D$ such that

$$
M(x) \operatorname{dist}(x, \Gamma)>2 k, \quad M(x) \geq M(y),
$$

and

$$
M(z) \leq 2 M(x) \quad \text { for all } z \in D \cap \bar{B}_{X}\left(x, k M^{-1}(x)\right) .
$$


Remarks 5.2. (a) In [28], Lemma 5.1 was used with $X=\mathbb{R}^{n}, \Sigma=\bar{\Omega}$ ( $\Omega$ domain of $\left.\mathbb{R}^{n}\right)$, and $\Gamma \subset \partial \Omega$, with the usual Euclidean distance $d(x, y)=|x-y|$. Here, we shall usually take $X=\mathbb{R}^{n+1}$ with the parabolic distance $d_{P}((x, t),(y, s))=|x-y|+|t-s|^{1 / 2}$, $D \subset \mathbb{R}^{n+1}$ and $\Gamma$ will be a part (or the whole of) the topological boundary of $D$ in $X$.

(b) If $\Sigma=\bar{\Omega} \times[0, T]$ and one knows that $M$ is bounded on $\bar{\Omega} \times[\varepsilon, T-\varepsilon]$ for each $\varepsilon>0$, then it is sufficient to apply Lemma 5.1 to the function $N(t)=\|M(\cdot, t)\|_{\infty}$ with $X=\mathbb{R}, \Sigma=[0, T]$ and $D=(0, T)$. However this need not be the case if one deals with a parabolic equation without boundary conditions (or in an unbounded spatial domain $\Omega$ ).

We turn to the proof of Theorem 3.1. We will assume that $u$ solves equation (1.2) in $D$, where $D$ is an arbitrary domain of $\mathbb{R}^{n+1}$ in the nonradial case, or $D=\Omega \times\left(T_{1}, T_{2}\right)$ with $-\infty \leq T_{1}<T_{2} \leq \infty$ and $\Omega$ a symmetric domain in the radial case. We will prove estimates (3.9) and (3.10) from Remark 3.4(e). We first consider the case $f(u)=u^{p}$.

Proof of Theorem 3.1 (ii). Assume that estimate (3.10) fails. Then, there exist sequences $D_{k}, u_{k},\left(y_{k}, \tau_{k}\right) \in D_{k}$, such that $u_{k}$ solves (1.1) in $D_{k}$ and the functions

$$
M_{k}:=u_{k}^{\frac{p-1}{2}}+\left|\nabla u_{k}\right|^{\frac{p-1}{p+1}}, \quad k=1,2, \ldots
$$

satisfy

$$
M_{k}\left(y_{k}, \tau_{k}\right)>2 k d_{P}^{-1}\left(\left(y_{k}, \tau_{k}\right), \partial D_{k}\right)
$$

where

$$
d_{P}((x, t),(y, s))=|x-y|+|t-s|^{1 / 2} .
$$

We will use Lemma 5.1 with $X=\mathbb{R}^{n+1}$, equipped with the parabolic distance $d_{P}$, $\Sigma=\Sigma_{k}=\bar{D}_{k}, D=D_{k}$, and $\Gamma=\partial D_{k}$, the topological boundary of $D_{k}$ in $X$. By this lemma, it follows that there exists $\left(x_{k}, t_{k}\right) \in D_{k}$ such that

$$
M_{k}\left(x_{k}, t_{k}\right) \geq M_{k}\left(y_{k}, \tau_{k}\right), \quad M_{k}\left(x_{k}, t_{k}\right)>2 k d_{P}^{-1}\left(\left(x_{k}, t_{k}\right), \partial D_{k}\right),
$$

and

$$
\begin{aligned}
& M_{k}(x, t) \leq 2 M_{k}\left(x_{k}, t_{k}\right), \quad \text { for all }(x, t) \in \mathbb{R}^{n+1} \\
& \text { such that } d_{P}\left((x, t),\left(x_{k}, t_{k}\right)\right) \leq k \lambda_{k},
\end{aligned}
$$

where

$$
\lambda_{k}=M_{k}^{-1}\left(x_{k}, t_{k}\right) .
$$

(Notice that (5.6) implies $d_{P}\left(\left(x_{k}, t_{k}\right), \partial D_{k}\right)>2 k \lambda_{k}$. Hence any $(x, t)$ satisfying the condition $d_{P}\left((x, t),\left(x_{k}, t_{k}\right)\right) \leq k \lambda_{k}$ in (5.7) is automatically contained in $D_{k}$.)

We now consider the nonradial and radial cases separately.

A. Nonradial Case. We rescale $u_{k}$ by setting

$$
v_{k}(y, s):=\lambda_{k}^{2 /(p-1)} u_{k}\left(x_{k}+\lambda_{k} y, t_{k}+\lambda_{k}^{2} s\right), \quad(y, s) \in \tilde{D}_{k},
$$


where

$$
\tilde{D}_{k}:=\left\{y \in \mathbb{R}^{n}:|y|<k / 2\right\} \times\left(-k^{2} / 4, k^{2} / 4\right) .
$$

The function $v_{k}$ solves

$$
\partial_{s} v_{k}-\Delta_{y} v_{k}=v_{k}^{p}, \quad(y, s) \in \tilde{D}_{k},
$$

Moreover,

$$
\left[v_{k}^{\frac{p-1}{2}}+\left|\nabla v_{k}\right|^{\frac{p-1}{p+1}}\right](0,0)=\lambda_{k} M_{k}\left(x_{k}, t_{k}\right)=1
$$

and

$$
\left[v_{k}^{\frac{p-1}{2}}+\left|\nabla v_{k}\right|^{\frac{p-1}{p+1}}\right](y, s) \leq 2, \quad(y, s) \in \tilde{D}_{k} .
$$

By using parabolic $L^{q}$ estimates, Schauder estimates and standard imbeddings, we deduce that some subsequence of $v_{k}$ converges in $C_{\text {loc }}^{2,1}\left(\mathbb{R}^{n} \times \mathbb{R}\right)$ to a (classical) solution $v \geq 0$ of $(1.1)$ in $\mathbb{R}^{n} \times \mathbb{R}$. Moreover, $\left[v^{\frac{p-1}{2}}+|\nabla v|^{\frac{p-1}{p+1}}\right](0,0)=1$ by $(5.10)$, so that $v$ is nontrivial, and $v, \nabla v$ are bounded, due to (5.11). This contradicts Theorem $\mathrm{A}$ and proves Theorem 3.1(ii) in the non-radial case.

B. Radial Case. In this case, there exist $-\infty \leq T_{1 k}<T_{2 k} \leq \infty, 0 \leq R_{1 k}<R_{2 k} \leq$ $\infty$ such that $D_{k}=\Omega_{k} \times\left(T_{1 k}, T_{2 k}\right)$, with $\Omega_{k}=\left\{x: R_{1 k}<|x|<R_{2 k}\right\}$ if $R_{1 k}>0$ and $\Omega_{k}=\left\{x:|x|<R_{2 k}\right\}$ if $R_{1 k}=0$.

Since $u_{k}$ is radial, we will write (without fearing confusion) $u_{k}=u_{k}(r, t)$ and $M_{k}=M_{k}(r, t)$, where $r=|x|$. Then $u_{k}$ solves the equation

$$
u_{t}-u_{r r}-\frac{n-1}{r} u_{r}=u^{p}
$$

for $(r, t) \in\left(R_{1 k}, R_{2 k}\right) \times\left(T_{1 k}, T_{2 k}\right)$. If $R_{1 k}=0$ (that is $\Omega_{k}$ is a ball or the whole of $\mathbb{R}^{n}$ ) then the function $u_{k}$ is a $C^{2,1}$ function defined on $\left[0, R_{2 k}\right) \times\left(T_{1 k}, T_{2 k}\right)$ satisfying $\partial_{r} u_{k}(0, t)=0, t \in\left(T_{1 k}, T_{2 k}\right)$. Since $u_{k}$ is radial, we may assume $x_{k}=\left(r_{k}, 0, \cdots, 0\right)$. Formula (5.7) then implies that

$$
M_{k}(r, t) \leq 2 M_{k}\left(r_{k}, t_{k}\right) \text { for all } r>0, t \in \mathbb{R} \text { such that }\left|r-r_{k}\right|+\left|t-t_{k}\right|^{1 / 2} \leq k \lambda_{k} .
$$

Next we distinguish two cases:

(a) the sequence $\left\{r_{k} / \lambda_{k}\right\}$ is bounded;

(b) the sequence $\left\{r_{k} / \lambda_{k}\right\}$ is unbounded.

In case (a) we may assume $r_{k} / \lambda_{k} \rightarrow \rho_{0} \geq 0$ as $k \rightarrow \infty$. Notice also that if $R_{1 k}>0$ then (5.6) implies

$$
r_{k}>r_{k}-R_{1 k} \geq d_{P}\left(\left(x_{k}, t_{k}\right), \partial D_{k}\right)>2 k \lambda_{k},
$$

which is not possible for $k$ large due to the boundedness of $\left\{r_{k} / \lambda_{k}\right\}$. Hence $R_{1 k}=0$ for $k$ sufficiently large and we also have $r_{k} / \lambda_{k}<k / 2$ for $k$ sufficiently large. For such $k$, set

$$
v_{k}(\rho, s):=\lambda_{k}^{2 /(p-1)} u_{k}\left(\lambda_{k} \rho, t_{k}+\lambda_{k}^{2} s\right), \quad(\rho, s) \in \tilde{D}_{k},
$$


where

$$
\tilde{D}_{k}:=(0, k / 2) \times\left(-k^{2} / 4, k^{2} / 4\right) .
$$

The function $v_{k}$ solves the equation

$$
v_{s}-v_{\rho \rho}-\frac{n-1}{\rho} v_{\rho}=v^{p}
$$

in $\tilde{D}_{k}, \partial_{\rho} v_{k}(0, s)=0$ for $|s|<k^{2} / 4$,

$$
\left[v_{k}^{\frac{p-1}{2}}+\left|\partial_{\rho} v_{k}\right|^{\frac{p-1}{p+1}}\right]\left(r_{k} / \lambda_{k}, 0\right)=1
$$

and

$$
\left[v_{k}^{\frac{p-1}{2}}+\left|\partial_{\rho} v_{k}\right|^{\frac{p-1}{p+1}}\right](\rho, s) \leq 2, \quad(\rho, s) \in \tilde{D}_{k} .
$$

As in the nonradial case, passing to the limit we obtain a nonnegative bounded solution $v$ of $(5.13)$ in $(0, \infty) \times \mathbb{R}$ satisfying $v_{\rho}(0, s)=0$ for all $s$ and

$$
\left[v^{\frac{p-1}{2}}+\left|\partial_{\rho} v\right|^{\frac{p-1}{p+1}}\right]\left(\rho_{0}, 0\right)=1,
$$

which contradicts Theorem B.

In case (b) we may assume $r_{k} / \lambda_{k} \rightarrow \infty$ as $k \rightarrow \infty$. Now we set

$$
v_{k}(\rho, s):=\lambda_{k}^{2 /(p-1)} u_{k}\left(r_{k}+\lambda_{k} \rho, t_{k}+\lambda_{k}^{2} s\right), \quad(\rho, s) \in \tilde{D}_{k},
$$

where

$$
\tilde{D}_{k}:=\left(-\min \left(r_{k} / \lambda_{k}, k / 2\right), k / 2\right) \times\left(-k^{2} / 4, k^{2} / 4\right) .
$$

Then $v_{k}$ solves the equation

$$
v_{s}-v_{\rho \rho}-\frac{n-1}{\rho+r_{k} / \lambda_{k}} v_{\rho}=v^{p}
$$

in $\tilde{D}_{k}$

$$
\left[v_{k}^{\frac{p-1}{2}}+\left|\partial_{\rho} v_{k}\right|^{\frac{p-1}{p+1}}\right](0,0)=1
$$

and

$$
\left[v_{k}^{\frac{p-1}{2}}+\left|\partial_{\rho} v_{k}\right|^{\frac{p-1}{p+1}}\right](\rho, s) \leq 2, \quad(\rho, s) \in \tilde{D}_{k} .
$$

Passing to the limit we obtain a nonnegative bounded solution of the equation

$$
v_{s}-v_{\rho \rho}=v^{p} \quad \text { in } \mathbb{R} \times \mathbb{R}
$$

satisfying

$$
\left[v^{\frac{p-1}{2}}+\left|\partial_{\rho} v\right|^{\frac{p-1}{p+1}}\right](0,0)=1
$$

which contradicts Theorem A with $n=1$. This concludes the proof of Theorem 3.1(ii). 
Next we deal with general $f$.

Proof of Theorem 3.1 (i). We first consider the nonradial case. Assume that estimate (3.9) fails. Keeping the same notation as in the proof of part (ii), we have sequences $D_{k}, u_{k},\left(y_{k}, \tau_{k}\right) \in D_{k}$, such that $u_{k}$ solves (1.2) in $D_{k}$ and

$$
M_{k}\left(y_{k}, \tau_{k}\right)>2 k\left(1+d_{P}^{-1}\left(\left(y_{k}, \tau_{k}\right), \partial D_{k}\right)\right)>2 k d_{P}{ }^{-1}\left(\left(y_{k}, \tau_{k}\right), \partial D_{k}\right) .
$$

Then, formulae (5.6)-(5.11) are unchanged except that the function $v_{k}$ now solves

$$
\partial_{s} v_{k}(y, s)-\Delta_{y} v_{k}(y, s)=f_{k}\left(v_{k}(y, s)\right):=\lambda_{k}^{\frac{2 p}{p-1}} f\left(\lambda_{k}^{\frac{-2}{p-1}} v_{k}(y, s)\right), \quad(y, s) \in \tilde{D}_{k}
$$

instead of (5.9), and that (since $M_{k}\left(x_{k}, t_{k}\right) \geq M_{k}\left(y_{k}, \tau_{k}\right)>2 k$ ) we also have

$$
\lambda_{k} \rightarrow 0, \quad k \rightarrow \infty
$$

Since $-C \leq f(s) \leq C\left(1+s^{p}\right), s \geq 0$, due to (3.1) (and $f$ being continuous), it follows that

$$
-C \lambda_{k}^{2 p /(p-1)} \leq f_{k}\left(v_{k}(y, s)\right) \leq C^{\prime}, \quad(y, s) \in \tilde{D}_{k} .
$$

By using parabolic $L^{q}$ estimates, standard imbeddings, and (3.1), we deduce that some subsequence of $v_{k}$ converges in $C_{\text {loc }}^{1,0}\left(\mathbb{R}^{n} \times \mathbb{R}\right)$ to a function $0 \leq v \in W_{\text {loc }}^{2,1, q}\left(\mathbb{R}^{n} \times \mathbb{R}\right)$, $1<q<\infty$, which satisfies $v_{s}-\Delta v \geq 0$. Moreover $\left[v^{\frac{p-1}{2}}+|\nabla v|^{\frac{p-1}{p+1}}\right](0,0)=1$ by $(5.10)$. Therefore, $v$ is nontrivial, and by the maximum principle there exists $s^{*} \in[-\infty, 0)$ such that $v(y, s)=0$ for any $y \in \mathbb{R}^{n}$ and $s \leq s^{*}, v(y, s)>0$ for any $y \in \mathbb{R}^{n}$ and $s>s^{*}$. Using assumption (3.1) again, we deduce that for each $(y, s) \in \mathbb{R}^{n} \times\left(s^{*}, \infty\right)$, $f_{k}\left(v_{k}(y, s)\right) \rightarrow \ell v^{p}(y, s)$ as $k \rightarrow \infty$. Consequently, $v$ is a solution of

$$
v_{s}-\Delta v=\ell v^{p}, \quad \text { in } \mathbb{R}^{n} \times\left(s^{*}, \infty\right)
$$

(and furthermore, $v$ and $\nabla v$ are bounded due to (5.11)). Now the uniqueness of the (continuous bounded) solution of the corresponding Cauchy problem guarantees $s^{*}=-\infty$. However, this contradicts Theorem A and proves Theorem 3.1(i) in the non-radial case.

The proof of Theorem 3.1(i) in the radial case is a straightforward modification of the above proof and of that of part (ii) in the radial case.

Proof of Theorem 4.1. It is obviously sufficient to prove assertion (i), i.e. the case $T<\infty$.

Assume that estimate (4.3) fails. Then, there exist sequences $T_{k} \in(0, \infty), u_{k}$, $y_{k} \in \Omega, s_{k} \in\left(0, T_{k}\right)$, such that $u_{k}$ solves (4.1) (with $T$ replaced by $T_{k}$ ) and the functions

$$
M_{k}:=u_{k}^{\frac{p-1}{2}}, \quad k=1,2, \ldots,
$$

satisfy

$$
M_{k}\left(y_{k}, s_{k}\right)>2 k\left(1+d_{k}^{-1}\left(s_{k}\right)\right)
$$


where $d_{k}(t):=\left(\min \left(t, T_{k}-t\right)\right)^{1 / 2}$. We will use Lemma 5.1 with $X=\mathbb{R}^{n+1}$, equipped with the parabolic distance $d_{P}$ defined by (5.5), $\Sigma=\Sigma_{k}=\bar{\Omega} \times\left[0, T_{k}\right], D=D_{k}=$ $\bar{\Omega} \times\left(0, T_{k}\right)$, and $\Gamma=\Gamma_{k}=\bar{\Omega} \times\left\{0, T_{k}\right\}$. Notice that

$$
d_{k}(t)=d_{P}\left((x, t), \Gamma_{k}\right), \quad(x, t) \in \Sigma_{k} .
$$

By Lemma 5.1, it follows that there exist $x_{k} \in \Omega, t_{k} \in\left(0, T_{k}\right)$ such that

$$
\begin{gathered}
M_{k}\left(x_{k}, t_{k}\right)>2 k d_{k}^{-1}\left(t_{k}\right), \\
M_{k}\left(x_{k}, t_{k}\right) \geq M_{k}\left(y_{k}, s_{k}\right)>2 k,
\end{gathered}
$$

and

$$
M_{k}(x, t) \leq 2 M_{k}\left(x_{k}, t_{k}\right), \quad(x, t) \in D_{k} \cap \tilde{B}_{k},
$$

where

$$
\tilde{B}_{k}:=\left\{(x, t) \in \mathbb{R}^{n+1} ;\left|x-x_{k}\right|+\left|t-t_{k}\right|^{1 / 2} \leq k \lambda_{k}\right\}
$$

and

$$
\lambda_{k}:=M_{k}^{-1}\left(x_{k}, t_{k}\right) \rightarrow 0 .
$$

Observe that for all $(x, t) \in \tilde{B}_{k}$, we have $\left|t-t_{k}\right| \leq k^{2} \lambda_{k}^{2}<d_{k}^{2}\left(t_{k}\right)=\min \left(t_{k}, T_{k}-t_{k}\right)$ by (5.17), hence $t \in\left(0, T_{k}\right)$. It follows that

$$
\left(\Omega \cap\left\{\left|x-x_{k}\right|<\frac{k \lambda_{k}}{2}\right\}\right) \times\left(t_{k}-\frac{k^{2} \lambda_{k}^{2}}{4}, t_{k}+\frac{k^{2} \lambda_{k}^{2}}{4}\right) \subset D_{k} \cap \tilde{B}_{k} .
$$

We now consider the nonradial and radial cases separately.

A. Nonradial Case. We rescale $u_{k}$ by setting

$$
v_{k}(y, s):=\lambda_{k}^{2 /(p-1)} u_{k}\left(x_{k}+\lambda_{k} y, t_{k}+\lambda_{k}^{2} s\right), \quad(y, s) \in \tilde{D}_{k},
$$

where

$$
\tilde{D}_{k}:=\left(\lambda_{k}^{-1}\left(\Omega-x_{k}\right) \cap\{|y|<k / 2\}\right) \times\left(-k^{2} / 4, k^{2} / 4\right) .
$$

The function $v_{k}$ solves

$$
\left.\begin{array}{rl}
\partial_{s} v_{k}-\Delta_{y} v_{k} & =f_{k}\left(v_{k}(y, s)\right), \quad(y, s) \in \tilde{D}_{k}, \\
v_{k} & \left.=0, \quad y \in \lambda_{k}^{-1}\left(\partial \Omega-x_{k}\right), \quad|y|<k / 2, \quad|s|<k^{2} / 4, \quad\right\}
\end{array}\right\}
$$

where

$$
f_{k}\left(v_{k}(y, s)\right):=\lambda_{k}^{\frac{2 p}{p-1}} f\left(\lambda_{k}^{\frac{-2}{p-1}} v_{k}(y, s)\right) .
$$

Moreover we have $v_{k}(0,0)=1$ and (5.18) implies

$$
v_{k} \leq C:=2^{\frac{2}{p-1}}, \quad(y, s) \in \tilde{D}_{k},
$$

hence

$$
-C \lambda_{k}^{2 p /(p-1)} \leq f_{k}\left(v_{k}(y, s)\right) \leq C, \quad(y, s) \in \tilde{D}_{k}
$$


due to (3.1), with $C>0$ independent of $k$. Let $\rho_{k}:=\operatorname{dist}\left(x_{k}, \partial \Omega\right)$. By passing to a subsequence, we may assume that either

$$
\rho_{k} / \lambda_{k} \rightarrow \infty
$$

or

$$
\rho_{k} / \lambda_{k} \rightarrow c \geq 0
$$

In case (5.24) holds, by using (5.21), (5.22), (5.23), (3.1), (5.19), interior parabolic estimates and standard imbeddings, and arguing as after formula (5.15), we deduce that some subsequence of $v_{k}$ converges in $C_{\mathrm{loc}}^{\alpha}\left(\mathbb{R}^{n} \times \mathbb{R}\right), 0<\alpha<1$, to a (classical) solution $v \geq 0$ of

$$
\partial_{s} v-\Delta_{y} v=\ell v^{p}, \quad x \in \mathbb{R}^{n}, \quad s \in \mathbb{R},
$$

with $v(0,0)=1$. This contradicts Theorem A.

In case (5.25) holds, denote $H_{c}:=\left\{y \in \mathbb{R}^{n} ; y_{1}>-c\right\}$. By performing a suitable orthogonal change of coordinates, (cf. [16] or [32] for example) using (5.19), (5.21), (5.22), (5.23), (3.1), interior-boundary parabolic estimates and standard imbeddings, we obtain a subsequence of $v_{k}$ which converges in $C_{\mathrm{loc}}^{\alpha}\left(\bar{H}_{c}\right), 0<\alpha<1$, to a (classical) bounded solution $v \geq 0$ of

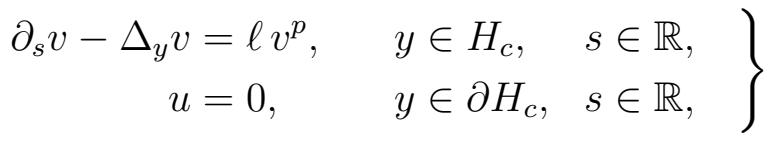

with $v(0,0)=1$ (hence $c>0$ ). This contradicts Theorem 2.1(i).

B. Radial Case. The end of the proof is a modification of the "nonradial" proof as in the case of Theorem 3.1. Since $\Omega$ is symmetric, there exist $0 \leq R_{1}<R_{2} \leq \infty$ such that $\Omega=\left\{x: R_{1}<|x|<R_{2}\right\}$ if $R_{1}>0$ and $\Omega=\left\{x:|x|<R_{2}\right\}$ if $R_{1}=0$. As in the proof of Theorem 3.1 we will write $u_{k}=u_{k}(r, t)$ and $M_{k}=M_{k}(r, t)$, where $r=|x|$. Then $u_{k}$ solves the equation

$$
u_{t}-u_{r r}-\frac{n-1}{r} u_{r}=f(u)
$$

in $\left(R_{1}, R_{2}\right) \times\left(0, T_{k}\right)$ and $u_{k} \in C^{2,1}\left(\left[0, R_{2}\right) \times(0, T)\right), \partial_{r} u_{k}(0, t)=0$ if $R_{1}=0$. By (5.18) and (5.20) we have

$$
\begin{aligned}
& M_{k}(r, t) \leq 2 M_{k}\left(r_{k}, t_{k}\right), \quad \text { for all }(r, t) \in\left(R_{1}, R_{2}\right) \times \mathbb{R} \\
& \text { such that }\left|r-r_{k}\right| \leq k \lambda_{k} / 2 \text { and }\left|t-t_{k}\right| \leq k^{2} \lambda_{k}^{2} / 4 .
\end{aligned}
$$

Next we distinguish two cases:

(a) the sequence $\left\{r_{k} / \lambda_{k}\right\}$ is bounded (this is possible only if $R_{1}=0$ );

(b) the sequence $\left\{r_{k} / \lambda_{k}\right\}$ is unbounded. 
In case (a) we may assume $r_{k} / \lambda_{k} \rightarrow \rho_{0} \geq 0$ as $k \rightarrow \infty$. Then $r_{k} / \lambda_{k}<k / 2$ for $k$ sufficiently large and we set

$$
v_{k}(\rho, s):=\lambda_{k}^{2 /(p-1)} u_{k}\left(\lambda_{k} \rho, t_{k}+\lambda_{k}^{2} s\right), \quad(\rho, s) \in \tilde{D}_{k},
$$

where

$$
\tilde{D}_{k}:=\left(0, \min \left\{k / 2, R_{2} / \lambda_{k}\right\}\right) \times\left(-k^{2} / 4, k^{2} / 4\right) .
$$

The function $v_{k}$ solves the equation

$$
\partial_{s} v_{k}-\partial_{\rho \rho}^{2} v_{k}-\frac{n-1}{\rho} \partial_{\rho} v_{k}=f_{k}\left(v_{k}(\rho, s)\right):=\lambda_{k}^{\frac{2 p}{p-1}} f\left(\lambda_{k}^{\frac{-2}{p-1}} v_{k}(\rho, s)\right)
$$

in $\tilde{D}_{k}, \partial_{\rho} v_{k}(0, s)=0$ for $|s|<k^{2} / 4$,

$$
v_{k}\left(r_{k} / \lambda_{k}, 0\right)=1, \quad v_{k}^{\frac{p-1}{2}}(\rho, s) \leq 2, \quad(\rho, s) \in \tilde{D}_{k} .
$$

Passing to the limit we obtain a nontrivial nonnegative bounded solution of

$$
v_{s}-v_{\rho \rho}-\frac{n-1}{\rho} v_{\rho}=\ell v^{p}, \quad \rho \in(0, \infty), s \in \mathbb{R}
$$

satisfying $v_{\rho}(0, s)=0$ for any $s$, which contradicts Theorem B.

In case (b) we may assume $r_{k} / \lambda_{k} \rightarrow \infty$ as $k \rightarrow \infty$. Now we set

$$
v_{k}(\rho, s):=\lambda_{k}^{2 /(p-1)} u_{k}\left(r_{k}+\lambda_{k} \rho, t_{k}+\lambda_{k}^{2} s\right), \quad(y, s) \in \tilde{D}_{k},
$$

where

$$
\tilde{D}_{k}:=\left\{\rho \in\left(\left(R_{1}-r_{k}\right) / \lambda_{k},\left(R_{2}-r_{k}\right) / \lambda_{k}\right):|\rho|<k / 2\right\} \times\left(-k^{2} / 4, k^{2} / 4\right) .
$$

Then $v_{k}$ solves

$$
\begin{aligned}
& \partial_{s} v_{k}-\partial_{\rho \rho}^{2} v_{k}-\frac{n-1}{\rho+r_{k} / \lambda_{k}} \partial_{\rho} v_{k}=f_{k}\left(v_{k}\right), \quad \text { in } \tilde{D}_{k}, \\
& v_{k}=0, \quad \rho \in\left\{\left(R_{1}-r_{k}\right) / \lambda_{k},\left(R_{2}-r_{k}\right) / \lambda_{k}\right\} \cap\{|\rho|<k / 2\},|s|<k^{2} / 4,
\end{aligned}
$$

where $f_{k}$ is as in (5.29). Moreover, $v_{k}(0,0)=1, v_{k}^{\frac{p-1}{2}} \leq 2$ in $\tilde{D}_{k}$, hence $\left|f_{k}\left(v_{k}\right)\right| \leq C_{1}$ in $\tilde{D}_{k}$.

Let $\eta_{k}:=\min \left\{R_{2}-r_{k}, r_{k}-R_{1}\right\}$. By passing to a subsequence, we may assume that either

$$
\eta_{k} / \lambda_{k} \rightarrow \infty
$$

or

$$
\eta_{k} / \lambda_{k} \rightarrow c_{0} \geq 0 .
$$

If (5.30) holds then passing to the limit we obtain a nontrivial nonnegative bounded solution of the equation

$$
v_{s}-v_{\rho \rho}=\ell v^{p}
$$


in $\mathbb{R} \times \mathbb{R}$, which contradicts Theorem $\mathrm{A}$.

If (5.31) holds then passing to the limit we obtain a nontrivial nonnegative bounded solution of the problem

$$
\begin{aligned}
v_{s}-v_{\rho \rho} & =\ell v^{p}, & & \rho \in H, s \in \mathbb{R}, \\
v & =0, & & \rho \in \partial H, s \in \mathbb{R},
\end{aligned}
$$

where either $H=\left(-c_{0}, \infty\right)$ or $H=\left(-\infty, c_{0}\right)$, which contradicts Theorem 2.1(i).

Proof of Theorem 4.2. The proof is similar to that of Theorem 4.1 in the nonradial case. The only difference is that we assume

$$
M_{k}\left(y_{k}, s_{k}\right)>2 k d_{k}^{-1}\left(s_{k}\right)
$$

instead of (5.16). Also we no longer have $\lambda_{k} \rightarrow 0$, but since $f_{k}\left(v_{k}\right)=v_{k}^{p}$ in (5.21) and $\Omega=\mathbb{R}_{+}^{n}$, the rest of the proof carries over.

Proof of Theorems 2.1(ii) and 2.3. Let $u$ be a nonnegative solution of (2.1), resp. (2.2). Fix $t_{0} \in \mathbb{R}$ and $\tau>0$. Applying Corollary 3.2, resp. Theorem 4.2, to the global solution $u_{t_{0}, \tau}:=u\left(\cdot, \cdot+t_{0}-\tau\right), t>0$, we obtain

$$
u\left(x, t_{0}\right)=u_{t_{0}, \tau}(x, \tau) \leq C(n, p) \tau^{-1 /(p-1)},
$$

for $x \in \mathbb{R}^{n}$, resp. $x \in \mathbb{R}_{+}^{n}$. Letting $\tau \rightarrow \infty$, we conclude that $u \equiv 0$.

Remarks 5.3. (a) Lemma 5.1 and the method of proof of Theorems 3.1 and 4.1 are a generalization of an idea of $\mathrm{B}$. $\mathrm{Hu}[20]$ (see also e.g. [7, 11, 23, 32, 25]). In those works, blow-up rate estimates and a priori bounds of global solutions were derived for various types of superlinear parabolic problems. By using a property similar to Lemma 5.1 (but concerning functions of the time variable only), it was shown that if a solution $u$ were violating a suitable estimate, then the function $M(t):=\|u(t)\|_{\infty}$ would satisfy $M(s) \leq 2 M\left(t_{k}\right)$ for all $s \in\left[t_{k}, t_{k}+k M^{1-p}\left(t_{k}\right)\right]$ and some sequence of times $t_{k}$. Then, by a rescaling argument similar to that used in the proof of Theorem 3.1, one was led to a contradiction with the corresponding nonexistence theorem. However, in all those works, the constants in the estimates depend on initial data. A main novelty in the present paper is the universality of the estimates and, in relation to this, the derivation of initial blow-up rates (and also of time decay and spatial singularity estimates). These improvements are made possible by Lemma 5.1 and by an appropriate application of the rescaling procedure.

(b) Let us justify Remark 4.3(b) concerning monotone in time solutions. If $u_{t} \geq 0$, then, in the proof of Theorem 4.1 (nonradial case), the rescaled solutions $v_{k}(y, s)$ satisfy $\partial_{s} v_{k} \geq 0$. Consequently, the bounded, positive solution $v(y, s)$ of the limiting problem (5.26) or (5.27) also satisfies $v_{s} \geq 0$. It is then easy to show that $v(y, s)$ converges, as $s \rightarrow+\infty$, to a (bounded) positive solution $w$ of the stationary problem $-\Delta w=w^{p}$, contradicting elliptic Liouville-type theorems for $p<p_{S}$ : see [14] or [8] 
for the whole space, [15] for the half-space. Thus we arrive at estimate (4.3). Now, since $u_{t} \geq 0$, we have

$$
u(x, t) \leq u(x, T / 2) \leq C(f, \Omega)\left(1+T^{-1 /(p-1)}\right)
$$

for $0<t<T / 2$, and

$$
u(x, t) \leq C(f, \Omega)\left(1+t^{-1 /(p-1)}+(T-t)^{-1 /(p-1)}\right) \leq C(f, \Omega)\left(1+(T-t)^{-1 /(p-1)}\right)
$$

for $T / 2 \leq t<T$, hence (4.5) holds. In the case $f(u)=u^{p}, \Omega=\mathbb{R}^{n},(4.5)$ implies (4.6) if $T=1$. For general $T>0$, (4.6) follows by an obvious scaling argument (replacing $u(x, t)$ by $\left.\tilde{u}(y, s):=T^{1 /(p-1)} u(\sqrt{T} y, T s)\right)$.

\section{Applications and generalizations}

Let us first mention an application of the universal estimate (4.3) to non-uniqueness for an initial-boundary value problem. In [4, Proposition 3] P. Baras proved a universal bound of the form

$$
u(x, t) \leq C(\delta), \quad t \in(\delta, T-\delta),
$$

for positive radial, radially decreasing solutions of (4.1) with $\Omega$ being a ball, $f(u)=u^{p}$ and $p<\min \left\{n /(n-2)_{+},(n+3) /(n-1)\right\}$. This result was used in order to prove the non-uniqueness of solutions of the problem

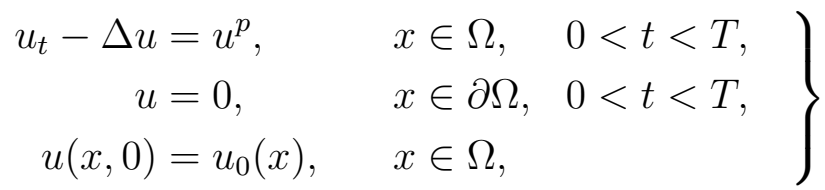

in the class

$$
X(q)=X(q, T):=C\left([0, T), L^{q}(\Omega)\right) \cap C^{2,1}(\bar{\Omega} \times(0, T)),
$$

$1 \leq q<n(p-1) / 2$. (Note that problem (6.1) is well posed in $X(q)$ for any $q>$ $n(p-1) / 2$.) Using Theorem 4.1 it is easy to modify the arguments of Baras in order to show the following assertion (cf. [4, Théorème 1]).

Proposition 6.1. Let $\Omega$ be a ball in $\mathbb{R}^{n}$ and $(n+2) / n<p<p_{S}$. Fix $r>n(p-1) / 2$ and assume that $u_{0} \in L^{r}(\Omega)$ is a positive radial, radially decreasing function. Let $T_{m}$ denote the maximal existence time of the corresponding solution $u_{m}$ of (6.1) in the class $X(r)$. Denote by $\|\cdot\|_{q}$ the norm in $L^{q}(\Omega)$. Then the following holds true.

(a) For any $T \in\left(0, T_{m}\right)$ there exists a function $u \geq u_{m}, u \neq u_{m}$, such that $u$ is a solution of (6.1) in the class $X(q, T)$ for any $q \in[1, n(p-1) / 2), u(\cdot, t)$ is radial and radially decreasing,

$$
\lim _{t \rightarrow 0}\left\|u(\cdot, t)-u_{0}\right\|_{q}=0 \quad \text { for any } q \in[1, n(p-1) / 2),
$$




$$
\begin{array}{ll}
\lim _{t \rightarrow 0}\|u(\cdot, t)\|_{q}=\infty & \text { for any } q>n(p-1) / 2, \\
\lim _{t \rightarrow T}\|u(\cdot, t)\|_{q}=\infty & \text { for any } q>n(p-1) / 2 .
\end{array}
$$

(b) If $\left\|u_{0}\right\|_{n(p-1) / 2} \leq c_{0}$ (where $c_{0}=c_{0}(\Omega, p)>0$ is a certain constant independent of $u_{0}$ ) then $T_{m}=\infty,\|u(\cdot, t)\|_{\infty} \rightarrow 0$ as $t \rightarrow \infty$, and there exists a function $u \geq$ $u_{m}, u \neq u_{m}$, such that $u$ is a solution of $(6.1)$ in the class $X(q, \infty)$ for any $q \in$ $[1, n(p-1) / 2), u(\cdot, t)$ is radial and radially decreasing, (6.2) and (6.3) are true and $\|u(\cdot, t)\|_{n(p-1) / 2} \geq c_{0}$ for any $t>0$.

We next consider some generalizations of our universal bounds. Similarly as in [28, Theorem 6.1], Theorems 3.1(i) and 4.1 can be easily generalized for nonlinearities of the form $f=f(x, t, u, \nabla u)$. More precisely, let $f$ be a Carathéodory function satisfying

$$
-C_{1}\left(1+s^{p_{1}}+|\xi|^{q_{1}}\right) \leq f(x, t, s, \xi) \leq C_{1}\left(1+s^{p}+|\xi|^{q}\right), \quad(x, t) \in D, s \geq 0, \quad \xi \in \mathbb{R}^{n},
$$

and, for all $(x, t) \in \bar{D}$,

$$
\lim _{s \rightarrow \infty, D \ni(z, \tau) \rightarrow(x, t)} s^{-p} f\left(z, \tau, s, s^{(p+1) / 2} \xi\right)=\ell(x, t) \in(0, \infty),
$$

uniformly for $\xi$ bounded. Here $q=2 p /(p+1), p_{1} \in(0, p), q_{1} \in(0, q)$ and if $D$ is unbounded then we assume that (6.4) also holds for $x=\infty$ and $t= \pm \infty$. Under these assumptions, Theorems 3.1(i) and 4.1 remain valid. Typical examples meeting these conditions are given by $f=u^{p} \pm|\nabla u|^{q}$, with $1 \leq q<2 p /(p+1)$. For previous results on (nonuniversal) blow-up rate estimates and a priori bounds for gradient-depending nonlinearities, see $[7,11,32,25,35]$ and the references therein.

Finally, as in the elliptic case (see [28]) we could also prove an analogue of Theorems 3.1 and 4.1 for systems of the form

$$
\begin{aligned}
& u_{t}-\Delta u=v^{p}, \\
& v_{t}-\Delta v=u^{q},
\end{aligned}
$$

where $p, q>1$ (cf. [28, Theorems 4.1-4.3 and Theorems 7.3, 7.5]). However, even in the elliptic case, optimal Liouville-type theorems are not known (except for the spatial dimensions $n \leq 3$ ). The situation in the parabolic case is much worse: the only nonexistence result for the above system in $\mathbb{R}^{n} \times \mathbb{R}$ is a consequence of the Fujita result [10] guaranteeing the nonexistence in $\mathbb{R}^{n} \times \mathbb{R}_{+}$for $\max (\alpha, \beta) \geq n$, where

$$
\alpha=\frac{2(p+1)}{p q-1} \quad \text { and } \quad \beta=\frac{2(q+1)}{p q-1} .
$$

Let us recall from [28] that an optimal condition for an elliptic Liouville-type theorem should be $\alpha+\beta>n-2$. It is a natural conjecture that this condition plays the same role in the parabolic case. 


\section{References}

[1] D. Andreucci and E. DiBenedetto, On the Cauchy problem and initial traces for a class of evolution equations with strongly nonlinear sources, Ann. Scuola Norm. Sup. Pisa Cl. Sci., Ser. IV 18 (1991), 363-441.

[2] D. Andreucci, M. A. Herrero and J. J. L. Velázquez, Liouville theorems and blow up behaviour in semilinear reaction diffusion systems, Ann. Inst. H. Poincaré, Analyse non linéaire 14 (1997), 1-53.

[3] D. Andreucci and A. Tedeev, Universal bounds at the blow-up time for nonlinear parabolic equations, Adv. Differ. Equations 10 (2005), 89-120.

[4] P. Baras, Non unicité des solutions d'une équation d'évolution non linéaire, Ann. Fac. Sci. Toulouse 5 (1983), 287-302.

[5] M.-F. Bidaut-Véron, Initial blow-up for the solutions of a semilinear parabolic equation with source term. Equations aux dérivées partielles et applications, articles dédiés à Jacques-Louis Lions, Gauthier-Villars, Paris, 1998, pp. 189-198.

[6] M.-F. Bidaut-Véron and L. Véron, Nonlinear elliptic equations on compact Riemannian manifolds and asymptotics of Emden equations, Invent. Math. 106 (1991), 489-539.

[7] M. Chlebík and M. Fila, From critical exponents to blowup rates for parabolic problems, Rend. Mat. Appl., Ser. VII 19 (1999), 449-470.

[8] W. Chen and C. Li, Classification of solutions of some nonlinear elliptic equations, Duke Math. J. 63 (1991), 615-622.

[9] N. Dancer, Some notes on the method of moving planes, Bull. Austral. Math. Soc. 46 (1992), 425-434.

[10] M. Escobedo and M.A. Herrero, Boundedness and blow up for a semilinear reaction-diffusion system, J. Differential Equations 89 (1991), 176-202.

[11] M. Fila and $\mathrm{Ph}$. Souplet, The blowup rate for semilinear parabolic problems on general domains, NoDEA Nonlinear Differ. Equations Appl. 8 (2001), 473-480.

[12] M. Fila, Ph. Souplet and F. Weissler, Linear and nonlinear heat equations in $L_{\delta}^{p}$ spaces and universal bounds for global solutions, Math. Ann. 320 (2001), 87-113.

[13] A. Friedman and J. B. McLeod, Blowup of positive solutions of semilinear heat equations, Indiana Univ. Math. J. 34 (1985), 425-447.

[14] B. Gidas and J. Spruck, Global and local behavior of positive solutions of nonlinear elliptic equations, Commun. Pure Appl. Math. 34 (1981), 525-598. 
[15] B. Gidas and J. Spruck, A priori bounds for positive solutions of a nonlinear elliptic equations, Commun. Partial. Differ. Equations. 6 (1981), 883-901.

[16] Y. Giga, A bound for global solutions of semilinear heat equations, Comm. Math. Phys. 103 (1986), 415-421.

[17] Y. Giga and R. Kohn, Characterizing blowup using similarity variables, Indiana Univ. Math. J. 36 (1987), 1-40.

[18] Y. Giga, S. Matsui and S. Sasayama, On blow-up rate for sign-changing solutions in a convex domain, Math. Methods Appl. Sci. 27 (2004), 1771-1782.

[19] A. Haraux and F. Weissler, Non-uniqueness for a semilinear initial value problem, Indiana Univ. Math. J. 31 (1981), 167-189.

[20] B. Hu, Remarks on the blowup estimate for solutions of the heat equation with a nonlinear boundary condition, Differ. Integral Equations 9 (1996), 891-901.

[21] O. Kavian, Remarks on the large time behaviour of a nonlinear diffusion equation, Ann. Inst. H. Poincaré, Anal. non linéaire 4 (1987), 423-452.

[22] H. A. Levine and P. Meier, A blowup result for the critical exponent in cones, Israel J. Math. 67 (1989), 129-136.

[23] Z. Lin, Blowup estimates for a mutualistic model in ecology, Electronic J. Qualit. Theory Differ. Equations 8 (2002) 1-14.

[24] J. Matos, Unfocused blow up solutions of semilinear parabolic equations, Discrete Contin. Dynam. Systems 5 (1999), 905-928.

[25] J. Matos and Ph. Souplet, Universal blow-up rates for a semilinear heat equation and applications, Adv. Differ. Equations 8 (2003), 615-639.

[26] F. Merle and H. Zaag, Optimal estimates for blowup rate and behavior for nonlinear heat equations, Comm. Pure Appl. Math. 51 (1998), 139-196.

[27] P. Poláčik and P. Quittner, A Liouville-type theorem and the decay of radial solutions of a semilinear heat equation, Nonlinear Anal., to appear.

[28] P. Poláčik, P. Quittner and Ph. Souplet, Singularity and decay estimates in superlinear problems via Liouville-type theorems. Part I: elliptic equations and systems, preprint (2005).

[29] P. Poláčik and E. Yanagida, On bounded and unbounded global solutions of a supercritical semilinear heat equation, Math. Ann. 327 (2003), 745-771.

[30] P. Quittner, A priori bounds for global solutions of a semilinear parabolic problem, Acta Math. Univ. Comenianae 68 (1999), 195-203. 
[31] P. Quittner, Universal bound for global positive solutions of a superlinear parabolic problem, Math. Ann. 320 (2001), 299-305.

[32] P. Quittner and Ph. Souplet, A priori estimates of global solutions of superlinear parabolic problems without variational structure, Discrete Contin. Dynam. Syst. 9 (2003), 1277-1292.

[33] P. Quittner, Ph. Souplet and M. Winkler, Initial blow-up rates and universal bounds for nonlinear heat equations, J. Differ. Equations 196 (2004), 316-339.

[34] Ph. Souplet, Sur l'asymptotique des solutions globales pour une équation de la chaleur semi-linéaire dans des domaines non bornés, C. R. Acad. Sci. Paris, Série I 323 (1996), 877-882.

[35] $\mathrm{Ph}$. Souplet, The influence of gradient perturbations on blow-up asymptotics in semilinear parabolic problems: a survey. Proceedings of the conference in honor of H. Amann (Zürich 2004), Progress in Nonlinear Differential Equations and Their Applications, Vol. 64, Birkhaüser, Basel, 2005, pp. 473-496. 